\title{
Mass Constituents of a Flat Lattice Multiverse: Conclusion from Similarity between Two Universal Numbers, the Rocksalt-Type 2D Madelung Constant and the Golden Mean
}

\author{
Hans Hermann Otto \\ Materialwissenschaftliche Kristallographie, Clausthal University of Technology, Clausthal-Zellerfeld, Germany \\ Email: hhermann.otto@web.de
}

How to cite this paper: Otto, H.H. (2018) Mass Constituents of a Flat Lattice Multiverse: Conclusion from Similarity between Two Universal Numbers, the Rocksalt-Type $2 D$ Madelung Constant and the Golden Mean. Journal of Modern Physics, 9, 1-13. https://doi.org/10.4236/jmp.2018.91001

Received: October 4, 2017

Accepted: January 5, 2018

Published: January 8, 2018

Copyright $\odot 2018$ by author and Scientific Research Publishing Inc. This work is licensed under the Creative Commons Attribution International License (CC BY 4.0).

http://creativecommons.org/licenses/by/4.0/ (c) (i) Open Access

\begin{abstract}
In fairly good agreement with the consensus range of dark energy to matter this ratio of the critical density is suggested to be connected with the golden mean $\varphi=0.6180339887$, yielding for dark energy to matter mass fractions $\Omega_{\Lambda} / \Omega_{m}=72.361 / 27.639=1+\varphi^{-1}=\varphi^{-2}=2.6180339$. Assuming the baryonic matter to be only $4.432 \%$, the ratio of matter to baryonic matter would be $\Omega_{m} / \Omega_{b}=6.236068=\varphi^{-3}+2=2 \varphi^{-2}+1$, and further the ratio of dark matter to baryonic one $\Omega_{d m} / \Omega_{b}=5.236068=\varphi^{-3}+1=2 \varphi^{-2}$. If one subtracts from the dark matter a contribution of antimatter with the same mass of baryonic matter, according to the antigravity theories of Villata respectively Hajdukovic, the remaining mass ratio would yield $\Omega_{r} / \Omega_{b}=4.236068=\varphi^{-3}=2 \varphi^{-2}-1$. Replacing the "Madelung" constant $\alpha$ of Villata's "lattice universe" by $\varphi$, one reaches again $1+\varphi$ as the ratio of the repulsive mass contribution to the attractive one. Assuming instead of a $3 D$ lattice a flat $2 D$ one of rocksalt type, the numerical similarity between the Madelung constant

$\alpha_{2 D}(\mathrm{NaCl})=1.6155426 \cdots$ and $\varphi^{-1}$ could not be just coincidence. The proposed scaling of the cosmological mass fractions with the square of the most irrational universal number $\varphi$ may indicate that the chaotic cosmological processes have reached a quite stable equilibrium. This may be confirmed by another, but similar representation of the mass constituents by the Archimedes' constant $\pi$, giving for $\Omega_{b}=(\pi-3) / \pi=0.04507$ respectively for the dark components $\Omega_{1-b}=3 / \pi=0.95493$. However, the intimate connection of $\varphi$ with its reciprocal may ignite the discussion whether our universe is intertwined with another universe or even part of a multiverse with the dark constituents contributed from there.
\end{abstract}




\section{Keywords}

Universal Numbers, Fractal Numbers, Golden Mean, Archimedes' Constant, Fibonacci Numbers, Madelung Constants, Sommerfeld's Fine Structure

Constant, Euler Number, Lattice Universe, Reciprocal Universe,

Cosmological Mass Fractions, Hubble Constant, Gyromagnetic Factor

\section{Introduction}

Since the discovery of the fivefold symmetry of quasicrystals, the present author as a crystallographer followed the appearance of this symmetry in nature. $\mathrm{He}$ was the first in Germany, who confirms the fivefold symmetry of quasicrystals by precise Guinier $X$-ray diffraction measurements [1]. Later, he described a Fibonacci superstructure of a pyroelectric bismuth sulfide iodide [2]. Even more, he applied the diffraction theory of a C60 buckyball to a cubic antiferromagnetic cuprate super-cage [3]. Finally, the research on high- $T_{\mathrm{c}}$ superconductors leads him to uncover the possible fractality and self-similarity of this class of compounds [4]. Familiar with the Fibonacci number sequence and the most irrational universal number of the golden mean, the motivation was obvious to deal with the Penrose universe, too.

Now follow this idea and consider two numerically comparable universal numbers, which may be suitable to combine theoretical approaches that deal with the secrets of our universe.

The first universal number that we highlight as golden mean $\varphi$ seems to people deeply familiar and induces the impression of perfect beauty. Inside, we suspect that it is something divine. Its secret is to mediate stability resulting from its most irrational character, which causes only particles as the center of gravity of vibrations with most irrational winding to survive [5] [6] [7]. In this version, $\varphi=(\sqrt{5}-1) / 2=0.618033 \cdots$ is used for the golden mean instead of $\varphi^{-1}=(\sqrt{5}+1) / 2=1.6180339887 \cdots$ as in previous versions to prevent confusion with upcoming publications. The second number is the Madelung constant $\alpha_{2 D}=1.6155426267 \cdots$ of a $2 D$ rocksalt-type lattice, familiar for crystallographers and material scientists that deal with ionic surfaces. It is found in Finch's monograph about Mathematical Constants [8]. Both numbers deviate from each other by merely $0.15 \%$. The numerical similarity of these numbers may suggest a connection of theoretical approaches that rely on these numbers. We ask for similarities between El Naschie's E-infinity theory [5] [6] and Villata's lattice universe [9], respectively Hajdukovic's theory of the gravitational polarization of the quantum vacuum [10].

\section{Explanation}

The impressive $E$-infinity $\left(\mathcal{\varepsilon}^{(\infty)}\right)$ theory of El Naschie [5] [6] formally explains the amount of dark matter based on the golden mean. However, Villata [9], Hajdu- 
kovic [10] and others (see further references given in [9] [10]) contributed the seminal idea that dark matter really does not exist and is merely the result of gravitational repulsion between matter and antimatter, once matter and antimatter have gravitational charges of opposite sign. Villata's cosmologic equation of antimatter gravity appears as a prediction of general relativity under $C P T$ boundary conditions and supports Hajdukovic's explanation of gravitational polarization of the quantum vacuum by only baryonic matter as today known. However, the $E$-infinity theory and the antigravity concept can be merged by adapting the two similar universal numbers used, namely the golden number and the Madelung constant $\alpha$ of Villata's "lattice universe". Herein matter particles and antimatter ones with the same mass, but different gravitational charges compose a lattice comparable with an ionic lattice such as $\mathrm{NaCl}$ in the electrostatic case. A result of Villata's cosmological equation [9] is that the ratio of the repulsive contribution to the attractive one would be $1+\alpha$, similar to the ratio of dark energy and matter. However, the similarity between both theoretical approaches mentioned is evident, if one compares, in contrast to the formerly used $3 D$ Madelung constant $\left(\alpha_{3 D}=1.7475645946 \cdots\right)$, the Madelung constant for a $2 D$ rocksalt-type lattice [8] [11] with the reciprocal of the golden mean

$$
\varphi^{-1}=1.6180339887 \cdots, \alpha_{2 D}(\mathrm{NaCl})=1.6155426267 \cdots
$$

The $\alpha_{2 \mathrm{D}}$ value is close to the quotient of two Fibonacci numbers, 21/13= $1.615385 \cdots$, and can be adapted to $\varphi^{-1}$ by only slight distortion of the square net along the two dimensions or by involving the third one to allow a quite flat curvature. For comparison, Smith [12] computed Madelung constants associated with the electrostatic energy of perfect one-component icosahedral quasicrystals with spherical acceptance volume to be $M_{\mathrm{qu}}=1.623$, and for the three-dimensional Penrose tiling $M_{\mathrm{pt}}=1.655$. Madelung constants for three-dimensional lattices respectively quasi-lattices are of course always greater than for corresponding two-dimensional lattices.

Tentatively, $\varphi$ instead of $\alpha$ may be used in Villata's cosmological equation [9]. Then the ratio of the repulsive contribution to the attractive one would be $1+\varphi^{-1}=2.6180339$. Consequently, the ratio between dark energy and matter would yield $72.36 \%$ to $27.64 \%$ of the critical density still in fairly good agreement with results of recent cosmological models [13] [14]. The ratio of matter to baryonic matter, when assuming the last one to be only $4.432 \%$, would be $\Omega_{m} / \Omega_{b}=6.236068=\varphi^{-3}+2=2 \varphi^{-2}+1$, and further the ratio of dark matter to baryonic one $\Omega_{d m} / \Omega_{b}=5.236068=\varphi^{3}+5=\varphi^{-3}+1=2 \varphi^{-2}$. If one subtracts from the dark matter a contribution of antimatter with the same mass of baryonic matter, according to the antigravity theories of Villata respectively Hajdukovic, the remaining mass ratio would yield $\Omega_{r} / \Omega_{b}=4.236068=\varphi^{-3}=2 \varphi^{-2}-1$. Finally, the ratio of dark energy to baryonic matter yields $\Omega_{\Lambda} / \Omega_{b}=\varphi^{-2}\left(2 \varphi^{-2}+1\right)=7 \varphi^{-2}-2$, and the overall density to the dark energy $\Omega_{o} / \Omega_{\Lambda}=100 / 72.361=1.38196=1+\varphi^{2}=3-\varphi^{-1}$, respectively. $\Omega_{b}$ is then reduced to 


$$
\Omega_{b}=\left(9 \varphi^{-2}-1\right)^{-1}=0.04432=1 / 22.56 \approx 22 / 496 \approx 4 / 89
$$

which may be compared with recent results of El Naschie's theory [7] [15], giving $\Omega_{b}=\varphi^{5} / 2=0.045085=k / 4=1 / 22.1803398$, where $k=0.1803398$ is the dimensional regularization order parameter of ' $t$ Hooft [15]. Subtracting our reciprocal baryonic mass fraction from El Naschie's value, one obtains

$$
22.5623064-22.1803398=0.3819660=\varphi^{2}
$$

Doing the same with the dark energy reciprocal fractions, one yields

$$
1.3819660-1.3708207=0.0111453=\varphi^{6} / 5
$$

Table 1 compares the proposed mass fractions with values of existing cosmological models respectively measurements.

Surprisingly, the overall mass density of the universe $\Omega_{o}$ can be calculated from the mass of matter as follows:

$$
\frac{\Omega_{o}}{\Omega_{m}}=\frac{\Omega_{o}}{\Omega_{d m}+\Omega_{b}}=3.618033989 \cdots=\varphi^{-2}+1
$$

With the quotient of the overall mass to the dark energy

$$
\frac{\Omega_{o}}{\Omega_{\Lambda}}=1.381966=1+\varphi^{2}
$$

we yield for the sum

$$
\frac{\Omega_{o}}{\Omega_{m}}+\frac{\Omega_{o}}{\Omega_{\Lambda}}=5
$$

The reciprocal quotients add up to

$$
\frac{\Omega_{m}}{\Omega_{o}}+\frac{\Omega_{\Lambda}}{\Omega_{o}}=1
$$

Now the reciprocal of the golden mean can be represented simply by the mean value of the root expressions:

$$
\frac{1}{2}\left\{\left(\frac{\Omega_{m}}{\Omega_{o}}+\frac{\Omega_{\Lambda}}{\Omega_{o}}\right)^{\frac{1}{2}}+\left(\frac{\Omega_{o}}{\Omega_{m}}+\frac{\Omega_{o}}{\Omega_{\Lambda}}\right)^{\frac{1}{2}}\right\}=\varphi^{-1}
$$

The geometrical mean gives

$$
\left\{\left(\frac{\Omega_{m}}{\Omega_{o}}+\frac{\Omega_{\Lambda}}{\Omega_{o}}\right) \cdot\left(\frac{\Omega_{o}}{\Omega_{m}}+\frac{\Omega_{o}}{\Omega_{\Lambda}}\right)\right\}^{\frac{1}{2}}=\sqrt{5}
$$

Table 1. Cosmological parameter values $\Omega$.

\begin{tabular}{cccccc}
\hline \multirow{2}{*}{ Primary Constituents } & \multicolumn{5}{c}{ Mass Fractions $\Omega$} \\
\cline { 2 - 6 } & \multicolumn{1}{c}{$\Lambda C D M$ model [13] } & WMAP mission [14] & $\varphi^{-2}$ scaling proposal \\
\hline baryonic matter & $0.0482 \pm 0.0016$ & 0.3067 & 0.046 & 0.04432 & 0.27639 \\
dark matter & $0.2585 \pm 0.0037$ & 0.24 & 0.23207 & \\
dark energy & $0.692 \pm 0.010$ & 0.714 & 0.72361 \\
\hline
\end{tabular}


The ratio of both until today invisible (dark) constituents, $\Omega_{\Lambda}$ and $\Omega_{d m}$, can also be described by solely fractal numbers (Fibonacci numbers respectively the golden mean):

$$
\frac{\Omega_{\Lambda}}{\Omega_{d m}}=\frac{21}{1 \times 2 \times 3}-\varphi^{2}=\varphi^{-2}+\frac{1}{2}=\left(7 \varphi^{-2}-2\right) / 2 \varphi^{-2}
$$

Table 2 summarizes the proposed scaling factors between the different primary mass constituents of the universe.

The proposed scaling of the cosmological mass fractions with the most irrational universal number $\varphi$ may indicate that the chaotic cosmological processes have reached a quite stable equilibrium state. Despite a supposed different evolution, the densities of dark energy and dark matter remain nearly equal for billion years.

Following cosmologists, which studied the microwave background and the distribution of galaxies, the geometry of the universe at the very large scales is close to flat. Of course, a three-dimensional lattice would better mimic our sensual experience of three spatial dimensions.

The inverse second power of the golden mean can be physically reasonably approximated, using the transcendent number of Sommerfelds fine structure constant $\alpha=0.0072973525664(17)$ [6] [16] [17]

$$
\varphi^{-2} \approx \frac{1}{2}(5 \alpha)^{-\frac{1}{2}}=2.61759
$$

Another universal fractal number, which is connected with the flat geometry of a quadratic Henon map, is $\delta_{1}=8.7210972$ [18] [19] [20]. Numerically, $\delta_{1}$ is approximately related to $\varphi^{-2}$ by

$$
\varphi^{-2}=\varphi^{-1}+1 \approx \frac{3}{5} \cdot \frac{\delta_{1}}{2}=2.61633
$$

The future will show whether this has any physical meaning.

However, the square of the Golden mean can also be properly approximated by [20]

Table 2. $\varphi^{2}$-based scaling factors between primary mass constituents $\Omega_{i}$ of the universe, $\varphi=(\sqrt{5}-1) / 2=0.61803398875 \cdots$.

\begin{tabular}{cccccc}
\hline $\boldsymbol{\Omega}_{\boldsymbol{i}}$ & $\boldsymbol{\Omega}_{\boldsymbol{o}}$ & $\boldsymbol{\Omega}_{\Lambda}$ & $\boldsymbol{\Omega}_{\boldsymbol{m}}$ & $\boldsymbol{\Omega}_{d m}$ & $\boldsymbol{\Omega}_{\boldsymbol{b}}$ \\
\hline $\boldsymbol{\Omega}_{\boldsymbol{o}}$ & 1 & $\varphi^{2}+1$ & $\varphi^{-2}+1$ & $3+\varphi^{-2} / 2$ & $9 \varphi^{-2}-1$ \\
$\boldsymbol{\Omega}_{\boldsymbol{\Lambda}}$ & $\left(\varphi^{-2}+1\right) / 5$ & 1 & $\varphi^{-2}$ & $1 / 2+\varphi^{-2}$ & $7 \varphi^{-2}-2$ \\
$\boldsymbol{\Omega}_{\boldsymbol{m}}$ & $\left(\varphi^{2}+1\right) / 5$ & $\varphi^{2}$ & 1 & $1+\varphi^{2} / 2$ & $2 \varphi^{-2}+1$ \\
$\boldsymbol{\Omega}_{d m}$ & $2 /\left(\varphi^{-2}+6\right)$ & $1 /\left(1 / 2+\varphi^{-2}\right)$ & $2 /\left(\varphi^{2}+2\right)$ & 1 & $2 \varphi^{-2}$ \\
$\boldsymbol{\Omega}_{b}$ & $1 /\left(9 \varphi^{-2}-1\right)$ & $1 /\left(7 \varphi^{-2}-2\right)$ & $1 /\left(2 \varphi^{-2}+1\right)$ & $1 / 2 \varphi^{-2}$ & 1 \\
\hline
\end{tabular}




$$
\varphi^{-2} \approx \frac{5}{3} \cdot \frac{\pi}{2}=2.61799388 \cdots,\left(\frac{5}{3} \cdot \frac{\pi}{2}\right)^{1 / 2}=1.6180216
$$

In a similar relation one can connect the Archimedes constant $\pi$ with $\varphi^{3}$ :

$$
\varphi^{3}=0.236067977 \approx \frac{5}{3}(\pi-3)
$$

Quoting El Naschie again [21], the number $5+\varphi^{3}=5.236067977$ represents the dimension of the fractal Kaluza-Klein spacetime. In contrast to our result, he yields for the ordinary matter fraction

$$
\Omega_{b}=\frac{\varphi^{3}}{5+\varphi^{3}}=0.045084
$$

Using equation (12), one gets

$$
\Omega_{b}=\frac{\pi-3}{\pi}=0.045070 .[20]
$$

This gives almost the same result. The alternative representation for the dark constituents (covering dark matter as well as dark energy) is convincingly simple [20]

$$
\Omega_{1-b}=\frac{5}{5+\varphi^{3}}=0.954915 \approx \frac{3}{\pi}=0.954930
$$

Interestingly, the factors applied in the equations 9 to 15 are always members of the Fibonacci numbers sequence.

Another number that is indirectly involved in the scaling factor between cosmological mass constituents in the anti-screening theory of Penner [22] is $e^{1}=$ $2.7182818 \cdots$, deviating only $3.8 \%$ from $\varphi^{-2}$ :

$$
\frac{e^{1}}{\varphi^{-2}}=\frac{2.718282}{2.618034}=1.0383
$$

Note that the Lambert $W$ function used in Penner's approach [22] is an analog to the golden mean for exponential functions, quoting the interesting contribution of Sherbon [23].

Furthermore, continuing still with Archimedes' respectively Euler numbers, our value for $\Omega_{b}$ can be approximated by

$$
\Omega_{b}=0.044322 \approx \pi^{-e}=0.044525 \cdots
$$

Combining Euler's number with ' $t$ Hooft renormalon $k$ one gets approximately

$$
e+\ln k \approx 1
$$

\section{An Intertwined "Reciprocal" Universe?}

The peculiar nature of the golden number is intimately connected with its reciprocal value. Especially we have, besides $\varphi-\varphi^{-1}=1$, the beautiful relation with respect to $\varphi^{2}$

$$
\left(\varphi^{-2}+1\right)^{-1}=\left(\varphi^{2}+1\right) / 5 \text { or equivalently }\left(\varphi^{2}+1\right)^{-1}=\left(\varphi^{-2}+1\right) / 5
$$


This quality of the squared golden mean suggests the calculation of reciprocal mass constituents of the universe. By renormalization of these reciprocal values, it is evident that the mass constituents simply interchange their values such that baryonic matter gets exactly the value of formerly dark energy and vice versa. Mathematically, this is always given for binary constituents of any $x$ and $1-x$, respectively. However, it is different for multi-component systems as shown in Table 3. Cosmological consequences may be well conceived.

Both "dark" constituents of the universe, introduced to overcome discrepancies between cosmological measurements and the theory, may have even more importance than discussed recently. Philosophically, there is scientific truth in the beauty of such symmetry. Are we living in a self-similar universe that itself is intertwined with its "reciprocal" counterpart? Is our universe part of a multiverse with a contribution of the dark constituents from there? One may see similarities of such a "universe" with Wolff s conjecture of the electron as a particle of nested highly coherent vibrations of most irrational frequency, a superposition of inwardly and outwardly extending spherical quantum waves [4] [24]. Since the author has studied self-similarity on quasi-crystals many years ago [1], the extracted results are once again stimulating.

Numerically, the reciprocal dark matter contribution (last column in Table 3) consists of two parts. Using symmetry arguments, it would be an obvious speculation to divide the dark matter also into two such proportions with a ratio of

$$
\frac{\Omega_{d m 1}}{\Omega_{d m 2}}=\frac{2.118034 \cdot \Omega_{b}}{3.118034 \cdot \Omega_{b}}=\frac{\left(\varphi^{-2}-\frac{1}{2}\right)}{\left(\varphi^{-2}+\frac{1}{2}\right)}=0.679285 \approx \frac{2}{3}
$$

Apparently, the following also results

$$
\Omega_{d m 2}-\Omega_{d m 1}=0.138197-0.093874=0.044322=\Omega_{b}
$$

Referring to the theory of El Naschie [7], about 5/22 of the overall density is attributed to dark matter. Now we split 5 into 2 and 3, respectively. Then we get mass fractions of $2 / 22=0.09091$ respectively $3 / 22=0.13636$, surprisingly near to the calculated values in Table 3. The representation of integers enhances the probability of this assumption to be realistic. Once assumed such diversification

\begin{tabular}{|c|c|c|c|}
\hline Primary Constituents & $\varphi^{2}$ Scaling & Reciprocal & Renormalized Reciprocal \\
\hline baryonic matter & 0.04432 & 22.56231 & 0.72361 \\
\hline \multirow[t]{2}{*}{ dark matter } & 0.23207 & 4.30899 & $0.13820+)$ \\
\hline & & $\left.2.92700^{*}\right)$ & 0.09387 \\
\hline dark energy & 0.72361 & 1.38197 & 0.04432 \\
\hline overall & 1.00000 & $\left.31.18034^{\star *}\right)$ & 1.00000 \\
\hline
\end{tabular}

Table 3. Reciprocal mass constituents of the universe.

$\left.{ }^{\star}\right)$ needed extension to optimize the renormalization such that $31.1802 / 22.5622=1.38197=1+\varphi^{2}$; ${ }^{*}$ ) $\left.31.18034=5\left(2 \varphi^{-2}+1\right) ;+\right)$ corresponds exactly to half the formerly $\Omega_{m}$ value (see Table 1 ). 
of dark matter, could it leave a fingerprint on the nature of the otherwise unseen particles of which it consists?

\section{Interpretation of the $\varphi^{2}$-Scaling}

The fractal Cantorian set theory [25] as a model for the dualism between a pre-quantum particle $P_{Q}$ and a pre-quantum wave $W_{Q}$, representing the zero set respectively its empty set boundary surrounding the pre-particle [26] [27], may deliver a modern interpretation of the $\varphi^{2}$ scaling. For the quantum wave, the bijective formula applied to the golden mean algebra yields the Hausdorff dimension [28] $d_{c}^{(n)}=\left(\varphi^{-1}\right)^{n-1}$ with the Urysohn-Menger [29] [30] topological dimension $n$, yielding for $n=-1$ the result $d_{c}^{-1}=\varphi^{2}$. Therefore, the empty set is represented by the bi-dimension $\operatorname{dim}\left(W_{Q}\right)=\left(n, d_{c}^{(n)}\right)=\left(-1, \varphi^{2}\right) \quad$ [26] [27], which is obviously the observed scaling behavior of the mass constituents of the Penrose-type intertwined multiverse. The zero set of the quantum particle $P_{Q}$, on the other hand, is represented by the bi-dimension $\operatorname{dim}\left(P_{Q}\right)=(0, \varphi)$, because for $n=0$ it applies $d_{c}^{0}=\left(\varphi^{-1}\right)^{0-1}=\varphi$.

Recently, El Naschie [31] quantified this approach further. He places the five-fold quasi-crystal symmetry in a Kaluza-Klein five-dimensional manifold and finds a five-dimensional zero set topological volume of $\varphi^{5}$. Then the surface of this volume yields an additive five-dimensional area equal to $5 \varphi^{2}$ that again represents the pre-quantum wave. In turn, he conjectured by an incredible conclusion that spacetime $(S p t)$ itself is a cobordism of the pre-quantum wave with a topological dimension of $n=-2$ and a Hausdorff dimension of $\varphi^{3}$, e.g., $\operatorname{dim}(S p t)=\left(-2, \varphi^{3}\right)$.

\section{Competition between $\varphi$ and $\pi$}

The circle number $\pi$, when considered as a fractal number (see Appendix for the continued fraction representation), could host a secret that the mankind has not yet solved. Noteworthy is the coincidental relationship between $\varphi$ and $\pi$ (corresponding to Equation (11)) that leads to an approximation of $\pi$, the Archimedes' constant (see also [32]):

$$
\frac{\Omega_{d m 2}}{\Omega_{b}}=\frac{6}{5} \varphi^{-2}=3.14164 \approx \pi
$$

We will apply this relation to find an alternative representation of the quantum-electrodynamic correction for the $g$-factor of the electron. First introduced by Schwinger [33], the quantum-electrodynamic correction for the $g$-factor of the electron should be $\alpha / \pi$ with Sommerfeld's fine structure constant $\alpha$. Combining the equations 9 and 22, respectively, one can express this correction as being proportional to the sixth power of the golden mean [34]:

$$
g_{e}=2+\frac{\varphi^{6}}{24}=2.0023220
$$

One can expand this formula by a second quadratic correction term to represent $g_{e}$ with higher accuracy, covering eight exact decimal places in com- 
parison with the highly precise experimental value [17]:

$$
g_{e}=2+\frac{\alpha}{\pi}-\frac{1}{2}\left(\frac{\alpha}{\pi}\right)^{2}=2+\frac{\varphi^{6}}{24}-\frac{1}{2}\left(\frac{\varphi^{6}}{24}\right)^{2}=2.002319307
$$

Tentatively, with the full series expansion for $\ln (1+x)$

$$
\ln (1+x)=x-\frac{x^{2}}{2}+\frac{x^{3}}{3}-\frac{x^{4}}{4} \pm \cdots, \quad \text { where } x=\frac{\varphi^{6}}{24}
$$

one finally obtains

$$
g_{e}=2+\ln (1.00232200)=2.002319312
$$

The very recently obtained experimental $g_{e}$ value as given in the NIST publication is [35]:

$$
g_{e}=2.00231930436182(52)
$$

At first glance, this result may be a surprise. However, as far as $\varphi$ is concerned, we follow Marek-Crnjac's explanation [36] and sum over all dimensions of infinite dimensional hierarchical Cantorian space-time instead of weighted trajectories of a system in the Feynman formulation of quantum mechanics. It is evident that the golden mean is one of the most important universal numbers which essentially affects our life and should influence our global perception and thinking. The explanation of the $g_{e}$ correction term by a Casimir radiative approach should be considered, correcting the sphere model of Rosencwaig by a factor of about two [37].

\section{A Short Comment on the Gravitational Anti-Screening Theory}

Let's leave the results presented in Table 2 aside Penner [22] published a gravitational anti-screening theory as an alternative to the standard $\Lambda C D M$ model that partly contrasts the approach of Hajdukovic [10]. His theory actually leads to the Baryonic Tully-Fisher Relation (BTFR) [38] and is consistent with a geometrically flat universe, respectively. Virtual particle-antiparticle pairs with the quality that like "charges" attract and unlike ones repel surround a baryonic mass in the vacuum and produce an anti-screening effect [22] that could explain the dark energy. For a baryonic mass fraction of $\Omega_{b}=0.0482$ [14], the model delivers a quite good overall density parameter of the universe of $\Omega_{o}=1.009$, when using the new value $H_{o}=(72.5 \pm 1.2) \mathrm{km} \cdot \mathrm{s}^{-1} \cdot \mathrm{Mpc}^{-1}$ for the Hubble constant, recently estimated from HOLiCOW experiments [39]. However, if one sets $\Omega_{o}=$ 1, the baryonic mass can be determined with Penner's density relation, recast as $\Omega_{b}=H_{o}^{2} \cdot R_{S C} /\left(4 \cdot g_{o}\right)$, where $g_{o}=(8.0 \pm 1.0) \times 10^{-11} \mathrm{~m} \cdot \mathrm{s}^{-2}$ is an empirical acceleration constant, derived from the $B T F R$, and $R_{\mathrm{SC}}=(89 \pm 16) \mathrm{Mpc}$ is half the separation between uniformly sized super-cluster centers [22]. One yields $\Omega_{b}=$ 0.0474 , whereas our value of $\Omega_{b}=0.0443$ could be reproduced (see Table 1) if the Hubble constant would be $70.1 \mathrm{~km} \cdot \mathrm{s}^{-1} \cdot \mathrm{Mpc}^{-1}$. The quotient of both Hubble constants mentioned is similar to that between the numbers $e^{1}$ and $\varphi^{-2}$. 


\section{Conclusions}

Cosmological mass fractions $\Omega_{\mathrm{i}}$ were tentatively scaled with the square of the most irrational universal number of the golden mean $\varphi$ still in the consensus range of recent observations and results. The similarity between this number and the Madelung constant $\alpha_{2 \mathrm{D}}$ of a $2 D$ rocksalt-type lattice, composed of matter and antimatter with gravitational charges of opposite sign, would yield the ratio of the repulsive contribution to the attractive one to be $1+\alpha \approx 1+\varphi^{-1}=\varphi^{-2}$, similar to the mass ratio of dark energy and matter. This actually delivers arguments for the lattice universe theory of Villata [9] and also for Hajdukovic's theory of the gravitational polarization of the quantum vacuum [10] respectively, the anti-screening approach of Penner [22]. Once more one should notice the intimate relation of the golden mean $\varphi$ with the Archimedes' constant $\pi$. Competing results for the mass constituents of the "universe" as discussed in this work may push forwards our view of the world with features supporting the concept of universes intimately penetrating each other. As anticipated by others, no Big Bang [40] or initial singularity [41] in the remote past is needed. The infinite extension of this system with a still immeasurable number of objects justifies once more the profitable application of number theory respectively set theory. In this sense, the found $\varphi^{-2}$-dependence of the cosmological mass fractions supports the "set-theoretical" interpretation given by El Naschie [31].

\section{References}

[1] Otto, H.H. and Ellner, M. (1987) Zeitschrift für Kristallographie 178, 178-179.

[2] Otto, H.H. (2015) World Journal of Condensed Matter Physics, 5, 66-77. https://doi.org/10.4236/wjcmp.2015.52010

[3] Otto, H.H. (2015) World Journal of Condensed Matter Physics, 5, 160-178. https://doi.org/10.4236/wjcmp.2015.53018

[4] Otto, H.H. (2016) World Journal of Condensed Matter Physics, 6, 244-260. https://doi.org/10.4236/wjcmp.2016.63023

[5] El Naschie, M.S. (2004) Chaos, Solitons and Fractals, 19, 209-236. https://doi.org/10.1016/S0960-0779(03)00278-9

[6] El Naschie, M.S. (2009) Chaos, Solitons and Fractals, 41, 2635-2646. https://doi.org/10.1016/j.chaos.2008.09.059

[7] El Naschie, M.S. (2014) Natural Sciences, 6, 1259-1265. https://doi.org/10.4236/ns.2014.616115

[8] Finch, S.R. (2003) Mathematical Constants. Cambridge University Press, New York, p. 76. https://doi.org/10.1017/CBO9780511550447

[9] Villata, M. (2013) Astrophysics and Space Science, 345, 1-10. https://doi.org/10.1007/s10509-013-1388-3

[10] Hajdukovic, D.S. (2011) Astrophysics and Space Science, 334, 215-218. https://doi.org/10.1007/s10509-011-0744-4

[11] Madelung, E. (1918) Physikalische Zeitschrift, 19, 524-533.

[12] Smith, A.P. (1990) Physical Review B, 42, 1189-1199. https://doi.org/10.1103/PhysRevB.42.1189 
[13] Ade, P.A.R., et al. (2014) Planck 2013. I. Overview of Products and Scientific Results. arXiv:1303.5062v2

[14] Wilkinson Microwave Anisotropy Probe 9-Year Results Released (2017) NASA Home Page.

[15] El Naschie, M.S. (2015) American Journal of Nano Research and Application, 3, $1-5$.

[16] Sommerfeld, A. (1919) Atombau und Spektrallinien. Friedrich Vieweg \& Sohn, Braunschweig.

[17] Aoyama, T., Hayakawa, M., Kinoshita, T. and Nio, M. (2012) Physical Review Letters, 109, Article ID: 111807. https://doi.org/10.1103/PhysRevLett.109.111807

[18] Hénon, M. (1976) Communications in Mathematical Physics, 50, 69-77. https://doi.org/10.1007/BF01608556

[19] Hellmann, R.H.G. (1980) Self-Generated Chaotic Behavior in Nonlinear Mechanics. In: Fundamental Problems in Statistical Mechanics, 5th Edition, E.G.D. Cohen, North-Holland Publishing Company, Amsterdam.

[20] Otto, H.H. (2017) Nonlinear Science Letters A, 8, 410-413.

[21] El Naschie, M.S. (2016) International Journal of Astronomy and Astrophysics, 6, 56-81. https://doi.org/10.4236/ijaa.2016.61005

[22] Penner, A.R. (2016) Astrophysics and Space Science, 361, 1-5.

[23] Sherbon, M.A. (2014) International Journal of Physical Research, 2, 1-9.

[24] Wolff, M. (1995) Galilean Electrodynamics, 6, 83-91.

[25] Cantor, G. (1932) Gesammelte Abhandlungen mathematischen und philosophischen Inhalts. Springer, Berlin.

[26] He, J.H. (2010) International Journal of Nonlinear Science and Numerical Simulations, 11, 1093-1095. https://doi.org/10.1515/IJNSNS.2010.11.12.1093

[27] Marek-Crnjac, L. (2011) Physics Research International, 2011, Article ID: 874302. https://doi.org/10.1155/2011/874302

[28] Hausdorff, F. (1918) Mathematische Annalen, 79, 157-179. https://doi.org/10.1007/BF01457179

[29] Urysohn, P. (1922) Compes Rendus, 175, 440-442.

[30] Menger, K. (1928) Dimensionstheorie. Verlag Leipzig und Berlin. https://doi.org/10.1007/978-3-663-16056-4

[31] El Naschie, M.S. (2017) Advances in Material Physics and Chemistry, 7, 347-352. https://doi.org/10.4236/ampc.2017.79027

[32] Dixon, R. (1991) The Story of Pi $(\pi)$. Mathographics 4.3, Dover, New York, 44-49 and 96-101.

[33] Schwinger, J. (1948) Physical Review, 73, 416-417. https://doi.org/10.1103/PhysRev.73.416

[34] Otto, H.H. (2017) Nonlinear Science Letters A, 8, 413-415.

[35] NIST (2017) The NIST Reference on Constants, Units, and Uncertainty. Gaithersburg.

[36] Marek-Crnjac, L. (2012) Quantum Gravity in Cantorian Space-Time. In: Sobreiro, R., Ed., Quantum Gravity, InTech, 87-100. https://doi.org/10.5772/37232

[37] Rosencwaig, A. (2006) A Casimir Approach for Radiative Self-Energy. 1-7. arXiv: hep-th/0606217v1

[38] McGaugh, S.S., Schombert, J.M., Bothun, G.D. and de Blok, W.J.G. (2000) Astro- 
physical Journal Letters, 533, L99-L102. https://doi.org/10.1086/312628

[39] Bonvin, V., et al. (2017) HOLiCOW V. New COSMOGRAIL Time Delays of HE0435-1223: $\mathrm{H}_{0}$ to $3.8 \%$ Precision from Strong Lensing in a Flat $\Lambda$ CDM Model. 1-19. arXiv:1607.01790v2 [astro-ph.CO]

[40] Bounias, M. and Krasnoholovets, V. (2005) The Universe from Nothing: A Mathematical Lattice of Empty Sets. 1-23. arXiv: physics/0309102v2 [physics.gen-ph]

[41] Hajdukovic, D.S. (2014) Physics of the Dark Universe, 3, 34-43. https://doi.org/10.1016/j.dark.2014.03.002 


\section{Appendix}

Continued Fraction Representation of the Golden Mean $\varphi$, the Fractal Kaluza-Klein Dimension $D_{\mathrm{KK}}$, the Approximated Circle Number $\pi$, the True Circle Number $\pi$, and the Euler Number $e$, respectively.

$$
\begin{aligned}
& \varphi=\frac{\sqrt{5}-1}{2}=\frac{1}{1+\frac{1}{1+\frac{1}{1+\cdots}}}=0.618033989 \ldots \\
& D_{K K}=5+\frac{1}{4+\frac{1}{4+\frac{1}{4+\cdots}}}=5+0.236067977 \cdots \\
& \pi \approx 3+\frac{3}{5} \cdot \frac{1}{4+\frac{1}{4+\frac{1}{4+\cdots}}}=3+0.1416407 \cdots \quad(\text { approximation of } \pi) \\
& \pi=3+\frac{1^{2}}{6+\frac{3^{2}}{6+\frac{5^{2}}{6+\cdots}}}=3+0.1415926 \cdots(\text { true circle constant }) \\
& e=2+\frac{1}{1+\frac{1}{2+\frac{2}{3+\frac{3}{4+\frac{4}{5+\frac{5}{6+\cdots}}}}}}=2.7182818284 \cdots \text { (Euler's number) }
\end{aligned}
$$

